\title{
DEVELOPMENNT OF A COMPOSITE SLAB BREAK-ELEMENT FOR THE ANALYSIS OF COMPOSITE FRAMES IN FIRE
}

\author{
Mohammadali Javaheriafif ${ }^{\mathrm{a}}$, Buick Davison ${ }^{\mathrm{b}}$, Ian Burgess ${ }^{\mathrm{c}}$ \\ ${ }^{\text {a,b,c }}$ University of Sheffield, Faculty of Civil and Structural Engineering, Sheffield, UK
}

\begin{abstract}
This research is intended to predict the inevitable through-depth crack development in a composite slab, across its area and in particular around its edges at large deflection. Based on previous work, a theoretical model has been proposed to simulate the local behaviour of slab beyond initial cracking. The model has been successfully implemented in the software VULCAN as a new line element. Comparisons between the existing theoretical model and FE modelling have shown that the proposed element provides a sufficient level of accuracy beyond initial cracking. However, further improvement is needed to enable a precise investigation of the local and global behaviour of composite slab systems, and the influence of through-depth cracking on the slab's performance.
\end{abstract}

Keywords: composite slab, crack, fracture, fire

\section{INTRODUCTION}

Different types of local failure cause different degrees of risk to a building. Progressive collapse can be the consequence of failure of a single load-carrying element. Joints are of particular importance, since the progressive collapse of a steel framed structure can be critically influenced by the performance of joints, especially under accidental extreme loading such as fire attack. The collapse of the twin towers of the World Trade Centre clearly illustrates the importance of joints in the overall performance of a structure, in which the collapse of the structure above the impact zone overloaded the structural joints, resulting in a sequence of failure downward to the ground (Bazant $\&$ Zhou 2002). Apart from the danger of progressive collapse in the structure, joint failure in fire is crucial, since any fracture of joints between beams and columns or beams and beams can lead to several undesirable effects such as spreading of fire to the upper floors through the gaps in cracked concrete slabs or lateral instability of a column resulting from a loss of connection to the beam and floor (Wang 2002).

Composite joints are one of the most common types of joint used in current structures. Extensive works have been done to investigate the performance of different types of bare steel connection over the last two decades, but little work has been done to investigate the influence of the composite slab on joint performance at elevated temperature.

A composite slab contributes to the rotational stiffness of the joint by means of its resistance to tensile force at the top surface of the slab due to hogging bending moment. The presence of the composite slab increases the lever arm within the joint, which consequently results in higher rotational stiffness. However, for the purpose of fire engineering design a joint should also be ductile enough to provide sufficient rotation capacity to the joint. According to the basic assumptions of current tensile membrane action analysis, the top surface of a slab panel at its edge support is mainly under tension as a result of hogging and tensile membrane stress. This can cause failure of the slab reinforcement across its edges (Bailey 2000). Fig.1 illustrates the distribution of compressive/tensile membrane force across a slab panel. 


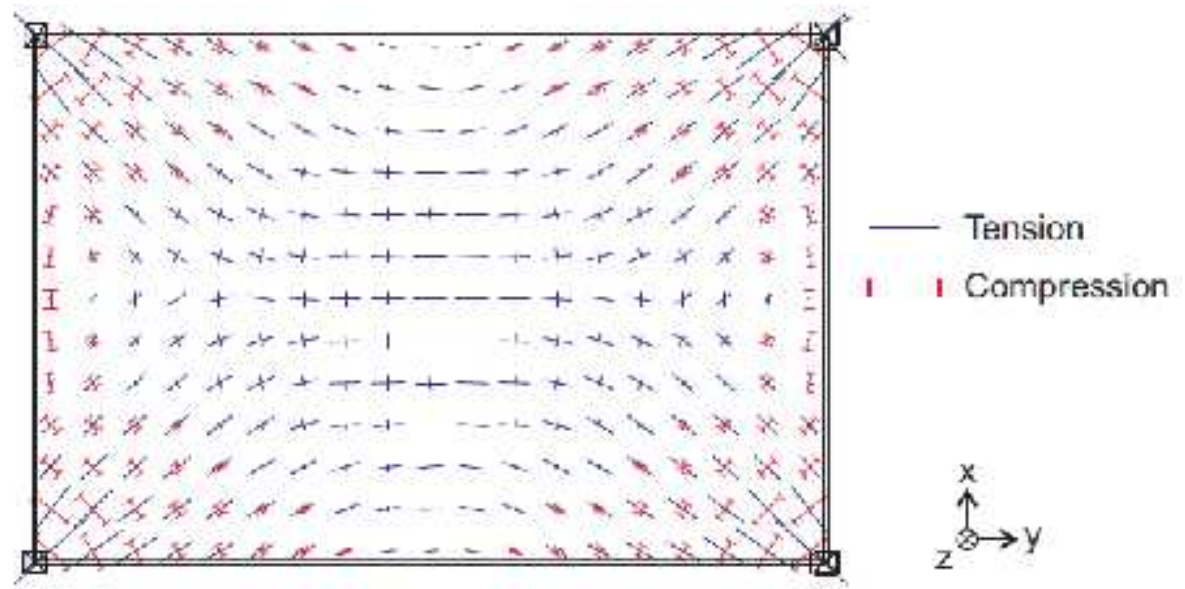

Fig. 1 Membrane forces of single slab [Stadler, 2012]

The top surface of the slab above the steel supporting beams cracks parallel to these beams as a result of distributed catenary tensile force due to the vertical deflection of the slab. Previous experimental results (Stadler 2012) show that a single crack occurred above the protected intermediate beams on different types of slab. The widths of these cracks were reported to be several millimetres, but this did not lead to the structural failure of the slabs. In general, even the failure of a slab at the edge beams results in no structural collapse, and structural stability is retained. However it has to be appreciated that, in fire resistance design, not only should a structure remain stable but it should also satisfy the integrity condition so that the fire does spread through openings in separating elements of the structure. Hence, it is important for the slab to meet not only the safety criteria "R" (load bearing capacity), but also "E" (compartment integrity), and "I" (insulation), thereby ensuring that no smoke, flames or unacceptable amounts of heat can penetrate to the floor above (Wang 2002).

\section{CRACK DEVELOPMENT THEORY}

A simplified model of the behaviour of the composite slab within the tension area has been produced, which represents the continuing concrete slab and its reinforcement in modelling beamcolumn and beam-beam connections. The concrete slab contributes to the overall performance of the structure through its composite action with the joint and connected steel beams in resisting the joint's horizontal and rotational movements. Therefore, any reasonable modelling of crack development over the steel beams becomes essential in order to investigate the influence of the slab on the overall performance of the composite joint. The development of pre-defined crack patterns in the concrete slab has been characterised using a new, so-called "Break Element".

\subsection{Characterisation of break element}

The cracking behaviour decisively depends on the characteristics of mesh reinforcement in the slab. Fig.2 illustrates a simplified stress-strain curve for cracking behaviour of a lightly-reinforced concrete slab, suggested by Stadler (2012). The first crack occurs when the concrete reaches its tensile strength, after which the total force has to be carried by the light rebar alone. Since the section is lightly reinforced the rebar has a lower tensile capacity than the force that was required to induce the first crack in the concrete. Therefore the reinforcement in the crack is unable to transfer enough force into the concrete to induce a second crack to form. Only one discrete crack can occur in the section before the reinforcement across the crack yields before finally rupturing when it reaches its ultimate strength.

The behaviour of the slab beyond its initial cracking is similar to what happens in a pull-out test conducted on a reinforced concrete section, in which the rebar embedded in the concrete subjected 
to axial tensile force can fail in one of the two traditionally acknowledged ways; pull-out failure or splitting failure.

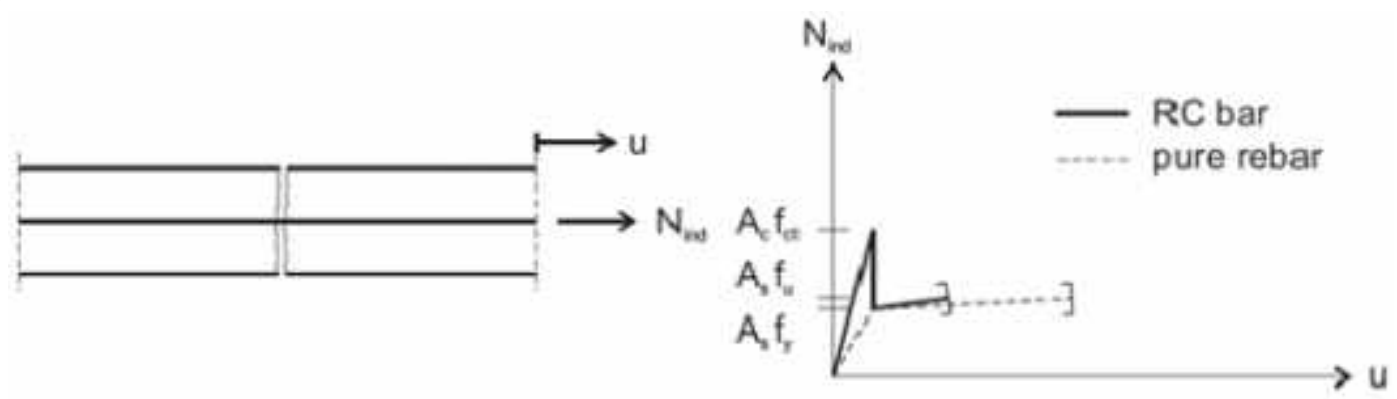

Fig. 2 Simplified crack behaviour of light RC member [Stadler,2012]

Therefore, it is possible to investigate the entire process of crack development in a slab by accurate modelling of the pull-out effect in RC members. Using this approach, the force-displacement response of the slab at a crack is determined, and has been used to characterise the break element. The effect of shear bond has been taken into account using an analytical model developed at the University of California-Berkeley (Sezen 2000). The model offers efficient and reliable calculation of reinforcement slip by assuming bi-uniform bond stress over the embedded length, which accounts for both elastic and plastic phases. Following this model, the displacement can be calculated by integrating the strain over the development length.

$\operatorname{sit} p=\int_{0}^{i_{1}+i_{d}} \varepsilon(x) d x$

$l_{a}=\frac{f_{s} \cdot a_{b}}{4 u_{b}} \quad, \quad l_{d}^{\prime}=\frac{\left(f_{z}-f_{y}\right) \cdot d_{b}}{4 u_{b}^{\prime}}$

Slip $=\frac{\varepsilon_{s} \cdot l_{d}}{2} \quad \varepsilon_{s} \leq \varepsilon_{y}$

Slip $=\frac{\varepsilon_{y} l_{d} d y}{2}+\frac{l_{d}^{\prime}}{2}\left(\varepsilon_{s}+\varepsilon_{y}\right) \quad \varepsilon_{s}>\varepsilon_{y}$

Where, $l_{a}$ and $l_{a}^{\prime}$ are the development lengths for the elastic and plastic zones of the bar, $\boldsymbol{u}_{b}$ and $\boldsymbol{u}_{b}^{\prime}$ are the uniform bond stresses for the elastic and plastic portions of the bar, which are approximated as $1 \sqrt{f^{\prime} c}$ and $0.5 \sqrt{f^{\prime} c}(\mathrm{MPa})$ respectively.

\section{NON-LINEAR PROCEDURE}

The approach is being implemented in the VULCAN software, which is capable of modeling geometrical non-linearity, considering non-linear material behaviour at elevated temperature. The composite steel-framed building is modelled as an assembly of finite beam-column, slab, connection and break elements.

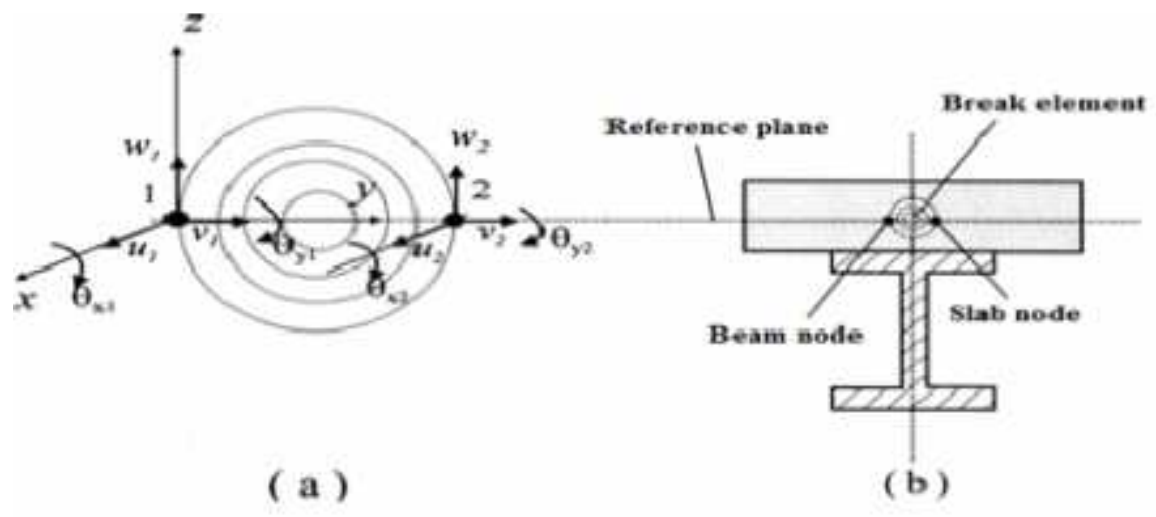

Fig. 3 Schematic of element's location in VULCAN 
It is assumed that the nodes of these different types of element are defined in a common reference plane, as shown in Fig.3. The software has the advantage of a combined static/dynamic solver, which makes it possible to trace the structural behaviour of a single member or frame from initial static response, through local failure or instability, to stable post-buckling. The composite joint is modelled using the existing two-dimensional component based model for bare steel connections, acting compositely with the 3D slab shell element through link elements representing shear studs.

\subsection{Break element}

The new element has been implemented in VULCAN with a multi-linear force-deformation characteristic. The first part of the curve represents an uncracked concrete section with negligible relative displacement. The second (elastic) phase starts as soon as concrete has cracked across the depth of slab, and the third phase is the plastic region where the embedded rebar is yielded and a reduced shear bond has been applied. The final part of the characteristic introduces the failure criterion for the break element, at which the ultimate strain of the assumed reinforcement is reached. Fig. 4 is a multi-linear schematic representation of the force-displacement relationship.

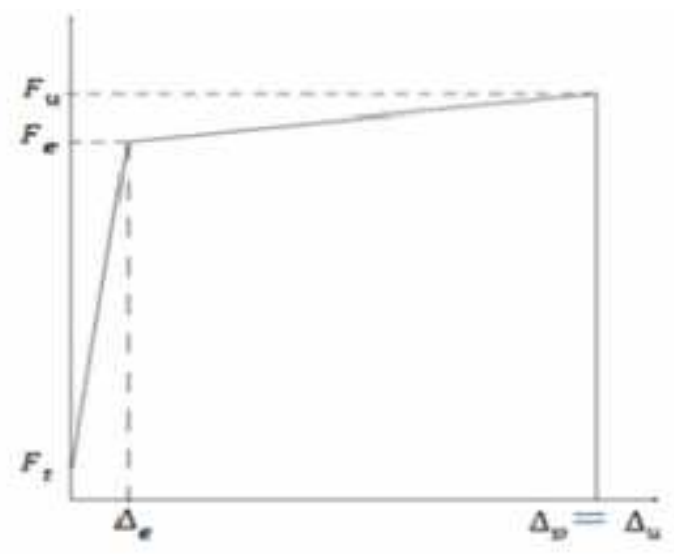

Fig. 4 Multi-linear force-deflection relationship - break element

The break element shown in Fig. 1(a) is being implemented in VULCAN as a specialized twonoded element of infinitesimally small length, which has three translational degrees of freedom $u, v, w$ and two rotational degrees of freedom $\theta_{x}, \theta_{y}$ at each node. Beyond the cracking of concrete in tension, the axial stiffness coefficient of $k_{1}$ or $k_{2}$ in the direction of the break element is obtained from the bond-slip relationship in Eqn. (1). The shear stiffness, $k_{3}$, is calculated using the slope deflection method, and the rotational stiffness are obtained by multiplying the lateral stiffness by the lever arm.

$$
k_{3}=\frac{12 E I}{L^{3}}, k_{4}=k_{2} \times r, k_{5}=k_{1} \times r
$$

Where, $E$ is the elastic modulus of the steel reinforcement, $I$ is the second moment of area and $r$ is the lever arm, which is the distance from the reference plane to the centre of beam element. The increment of internal force $\Delta F_{i}$ for each degree of freedom can be related to the increment of slip, $\Delta U_{i}$ by the tangent stiffness relationship.

$$
\Delta F_{i}=k_{i}, \Delta U_{i}
$$

The break element is assumed to be fairly rigid before the appearance of the crack at the top surface of the concrete floor with a stiffness similar to that of the adjacent uncracked slab element. Break elements are located across the floor area and at the perimeter nodes of every slab element across the slab area. This will enable a more accurate investigation of crack development within a slab panel. Once concrete at the top surface cracks following fracture of break elements the dynamic solver can temporarily be activated to search for the next re-stabilization. After re-stabilizing, the analysis continues using the static solver. Fig.5 illustrates a typical layout of composite floor structure modelled in VULCAN, with break elements between slab and beam nodes. 


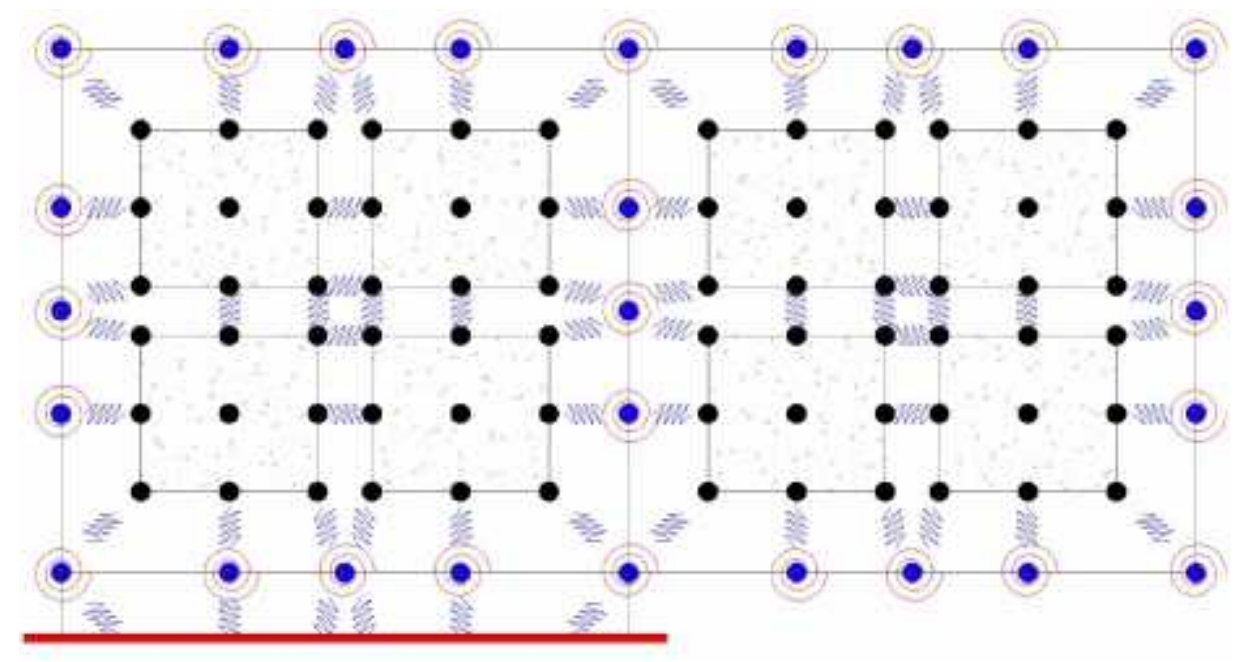

Fig. 5 Possible locations of break element in Vulcan

\section{VALIDATION AGAINST THEORETICAL MODEL}

The break element has been tested and validated against the standard pull-out test $(10 \mathrm{~cm}$ of embedded rebar, As $=50.2 \mathrm{~mm}^{2}$ ) using the validated theoretical bond-slip model proposed by Sezen (2000).

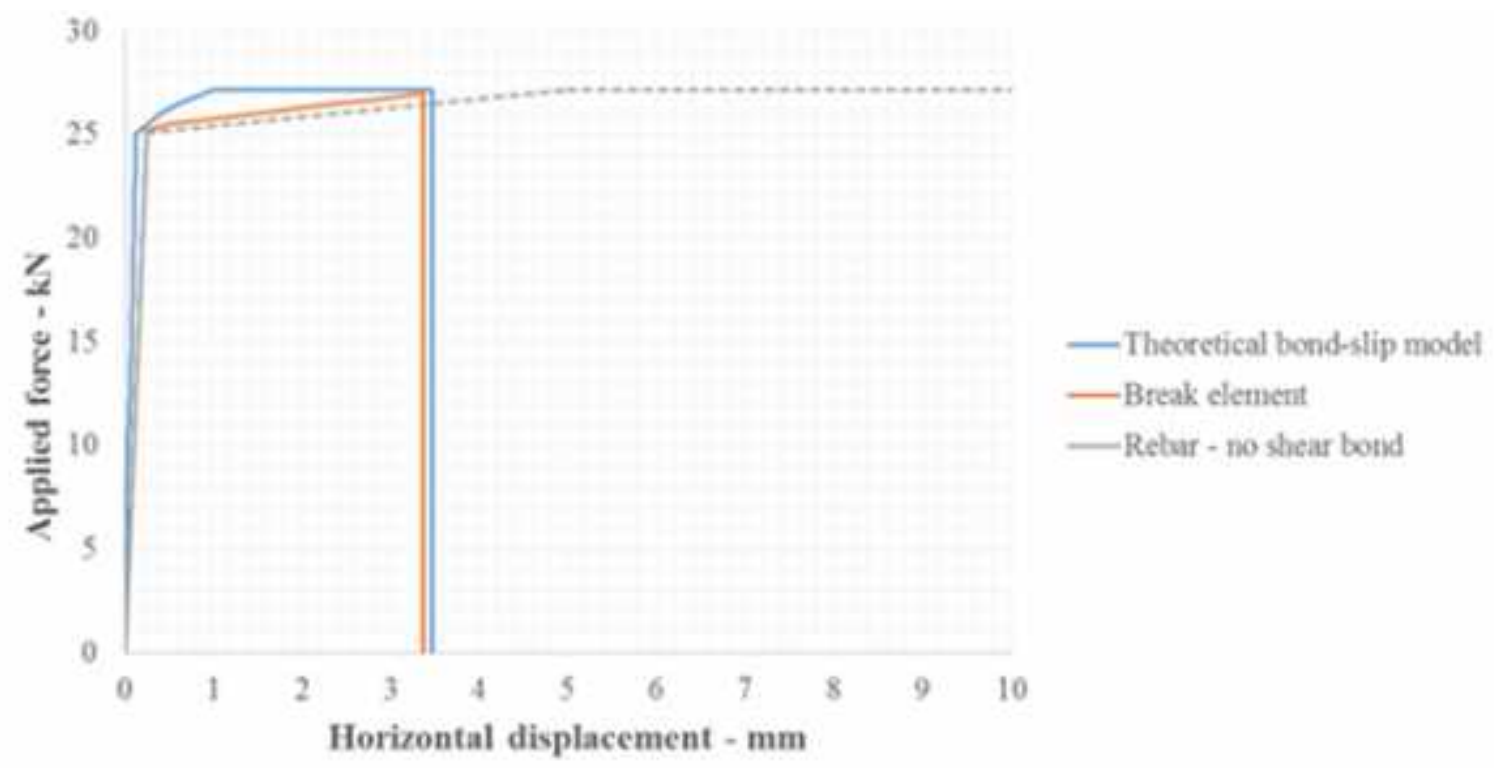

Fig. 6 Comparison of lateral displacements - pull out test

The new element has also been tested in bending. One break element was attached to a steel beam at each end, fixed to boundary conditions. Figs. 8 and 9 respectively illustrate the vertical displacement of the beam compared to the lateral displacement of the break element due to bending and the internal forces of break elements against applied load.

$$
6000 \mathrm{~mm}
$$

$13 \mathrm{~mm}$

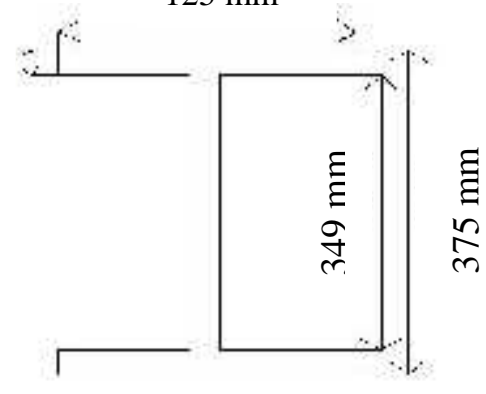

Fig. 7 Boundary condition \& Cross section - Beam 


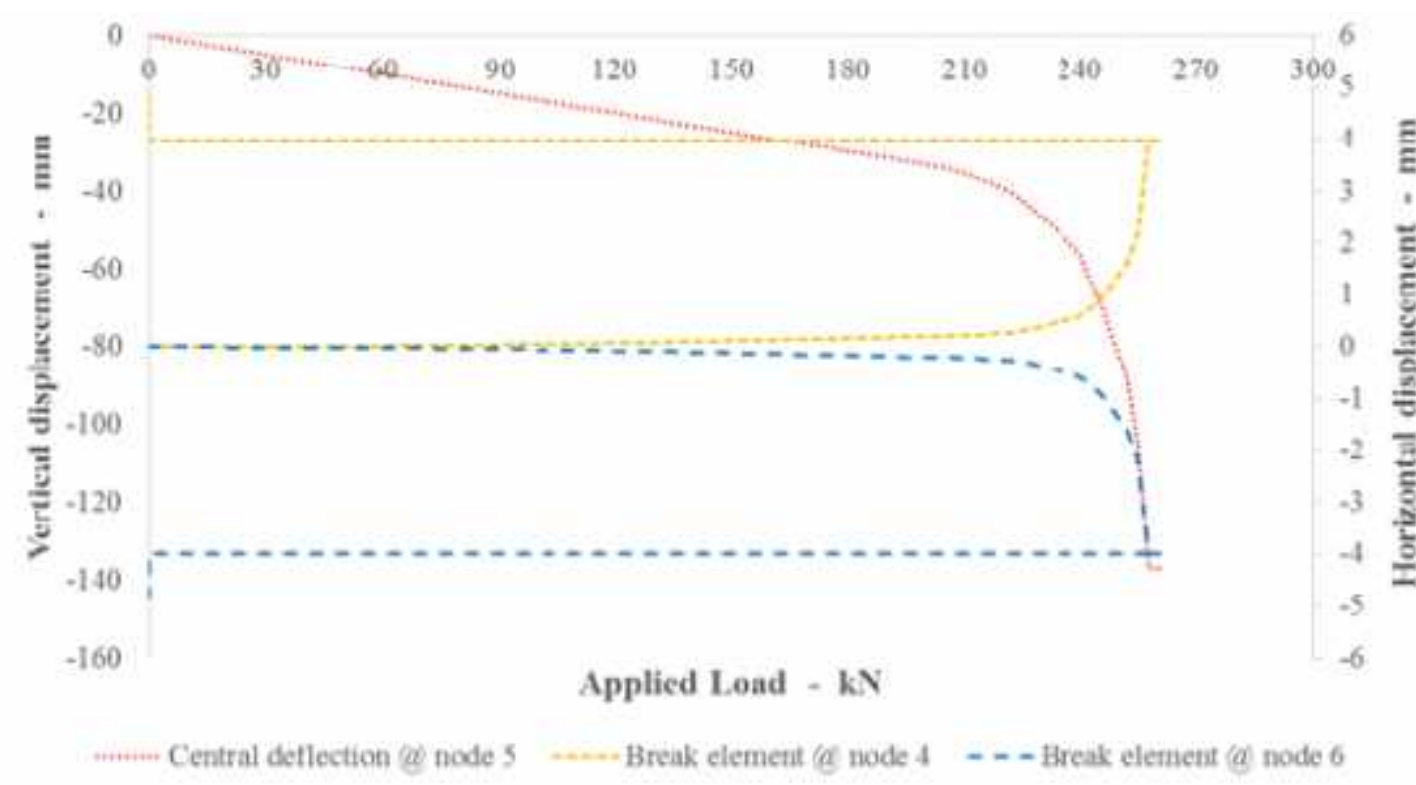

Fig. 8 Comparison of lateral/vertical displacements due to bending

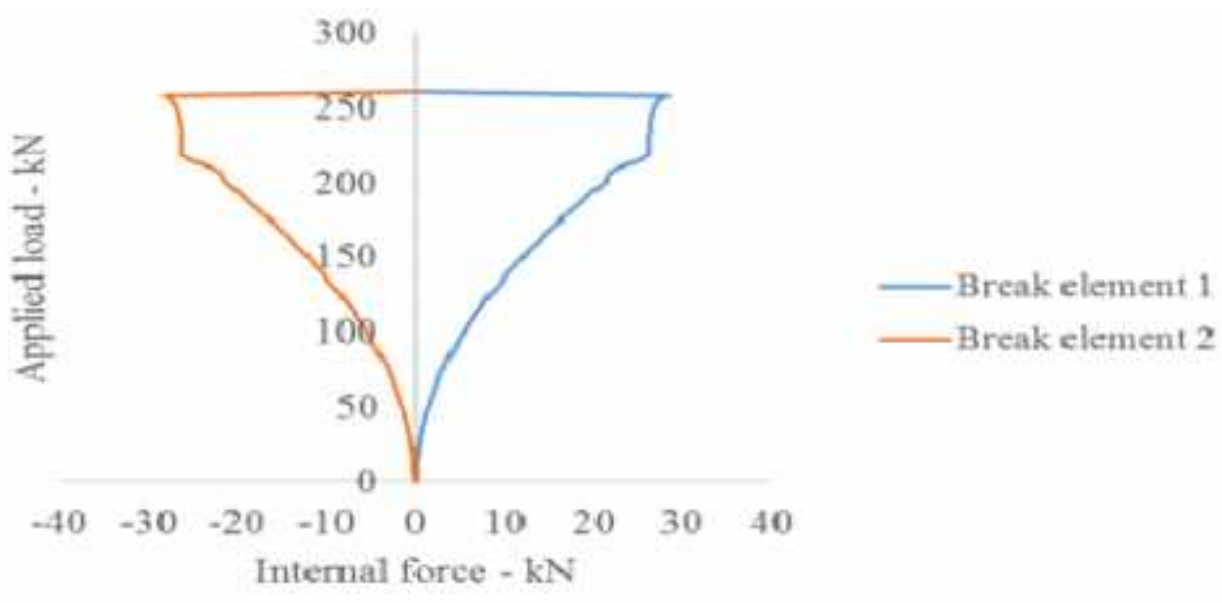

Fig. 9 Internal force vs applied force - break element

\section{CONCLUSIONS}

The proposed model has been implemented in VULCAN, seen to detect important structural effects and to produce logical results. The element has been validated against the theoretical model. Since there is currently insufficient experimental data it is necessary to rely on the structural logic of the predicted behaviour. The next step of the work is to investigate the influence of the occurrence of cracks on the overall performance of a slab using break elements.

\section{REFERENCES}

Bailey, C., 2000. The structural behaviour of steel frames with composite floorslabs subject to fire: Part 1: Theory. The structural engineer, 78(11), pp.19-27.

Bazant, Z.. \& Zhou, Y., 2002. Why did the World Trade Centre collapse? - Simple analysis. Journal of Mechanics, ASCE, (1), pp.2-6.

Sezen, H., 2000. Seismic Behavior and Modeling of Reinforced Concrete Building Columns. University of California, Berkeley.

Stadler, M., 2012. Design of Composite Slab Systems in Case of Fire Using Simplified Finite Element Analyses. Technische Universität München.

Wang, Y.C., 2002. Steel and composite structures : behaviour and design for fire safety, Spon, London. 